\title{
Special issue: The contributions of Erio Camporesi
}

\author{
Chayanard Phukhamsakda ${ }^{1}$ Nalin N. Wijayawardene ${ }^{1,11} \cdot$ Hiran A. Ariyawansa, ${ }^{1,3} \cdot$ Indunil C. Senanayake ${ }^{1,4}$. \\ Wen-Jing $\mathrm{Li}^{1,7}$. Dhanushka N. Wanasinghe ${ }^{1,2,5,6} \cdot$ Rungtiwa Phookamsak ${ }^{1,2,5,6} \cdot$ Qing Tian $^{1,7}$. \\ Dinushani A. Daranagama ${ }^{1,12} \cdot$ Kasun M. Thambugala ${ }^{1,8}$. Saowaluck Tibpromma ${ }^{1,2,5,6}$ - Anusha H. Ekanayaka ${ }^{1,2}$. \\ Marc Stadler $^{10} \cdot$ Kevin D. Hyde ${ }^{1,2,7,9}$
}

Published online: 23 March 2020

(c) MUSHROOM RESEARCH FOUNDATION 2020

We are proud to publish a special issue of Fungal Diversity in honour of the contributions made by Erio Camporesi, who has promoted mycological research as a prodigious amateur mycologist and collector of fungi. The special issue includes Fungal Diversity notes 11, with many taxa named in Erio's honour and a monograph of hyaline-spored Coelomycetes, both incorporating many of Erio's collections.

Erio obtained a certificate of accountancy in 1982 and worked as an accountant at the express courier company, TNT Global Express SPA. He was always interested in collecting microfungi, mushrooms and toadstools and in doing so this passion has occupied much of his spare time. His first collection in 1984 was of a mushroom, Suillellus luridus ( $\equiv$ Boletus luridus). Erio was particularly interested in microfungi associated with plants. He started to

Chayanard Phukhamsakda

chayanard91@gmail.com

1 Center of Excellence in Fungal Research, Mae Fah Luang University, Chiang Rai 57100, Thailand

2 Key Laboratory for Plant Diversity and Biogeography of East Asia, Kunming Institute of Botany, Chinese Academy of Sciences, Kunming 650201, Yunnan, People's Republic of China

3 Department of Plant Pathology and Microbiology, College of Bio-Resources and Agriculture, National Taiwan University, Taipei, Taiwan

4 Shenzhen Key Laboratory of Microbial Genetic Engineering, College of Life Sciences and Oceanography, Shenzhen University, Shenzhen 518060, People's Republic of China

5 East and Central Asia Regional Office, World Agroforestry Centre (ICRAF), Kunming 650201, Yunnan, People's Republic of China

6 Centre for Mountain Futures (CMF), Kunming Institute of Botany, Kunming 650201, Yunnan, People's Republic of China collaborate with Kevin D. Hyde through the AscoFrance website (http://www.ascofrance.com). The first fungal specimen to be loaned to Kevin D. Hyde was Dothidella ulmi. Since then, Erio has sent thousands of herbarium specimens for study. Erio's mother, Maria Ghetti, who accompanied Erio on many forays (Fig. 1), died on 21 April 2012 leaving Erio to care for his aging father, Nello Camporesi, who also accompanied Erio on forays and died on 24 February 2019 (Fig. 1). Erio retired from his permanent accounting job in 2013 and, thereafter, his interest in mycology became a top priority. He also had a talent for painting and many of his paintings incorporated fungi in imaginative ways (Fig. 2).

Italian mycology has an exceptional history with the early contributions by Giuseppe De Notaris (1805-1877), Vincenzo de Cesati (1806-1883) and Pier Andrea Saccardo (1845-1920) who greatly contributed to the fungal

Mushroom Research Foundation, 128 M.3 Ban Pa Deng T. Pa Pae, A. Mae Taeng, Chiang Mai 50150, Thailand

8 Industrial Science and Management (International Program), Faculty of Science and Technology, Thammasat University (Rangsit Center), Klong Luang 12121, Pathumthani, Thailand

9 Department of Biology, Faculty of Science, Chiang Mai University, Chiang Mai 50200, Thailand

10 Department of Microbial Drugs, Helmholtz Centre for Infection Research and German Centre for Infection Research (DZIF), Partner Site Hannover/Braunschweig, Inhoffenstrasse 7, 38124 Brunswick, Germany

11 Center for Yunnan Plateau Biological Resources Protection and Utilization, College of Biological Resource and Food Engineering, Qujing Normal University, Qujing 655011, Yunnan, People's Republic of China

12 Department of Plant and Molecular Biology, Faculty of Science, University of Kelaniya, Sri Lanka, Kelaniya, Sri Lanka 
Fig. 1 a Erio Camporesi. b At home with his compound microscope. c, e Erio Camporesi and Prof. Kevin D. Hyde at Italy in 2017. d Erio's parents, Maria Ghetti and Nello Camporesi during a collecting trip in Italy. Used with permission
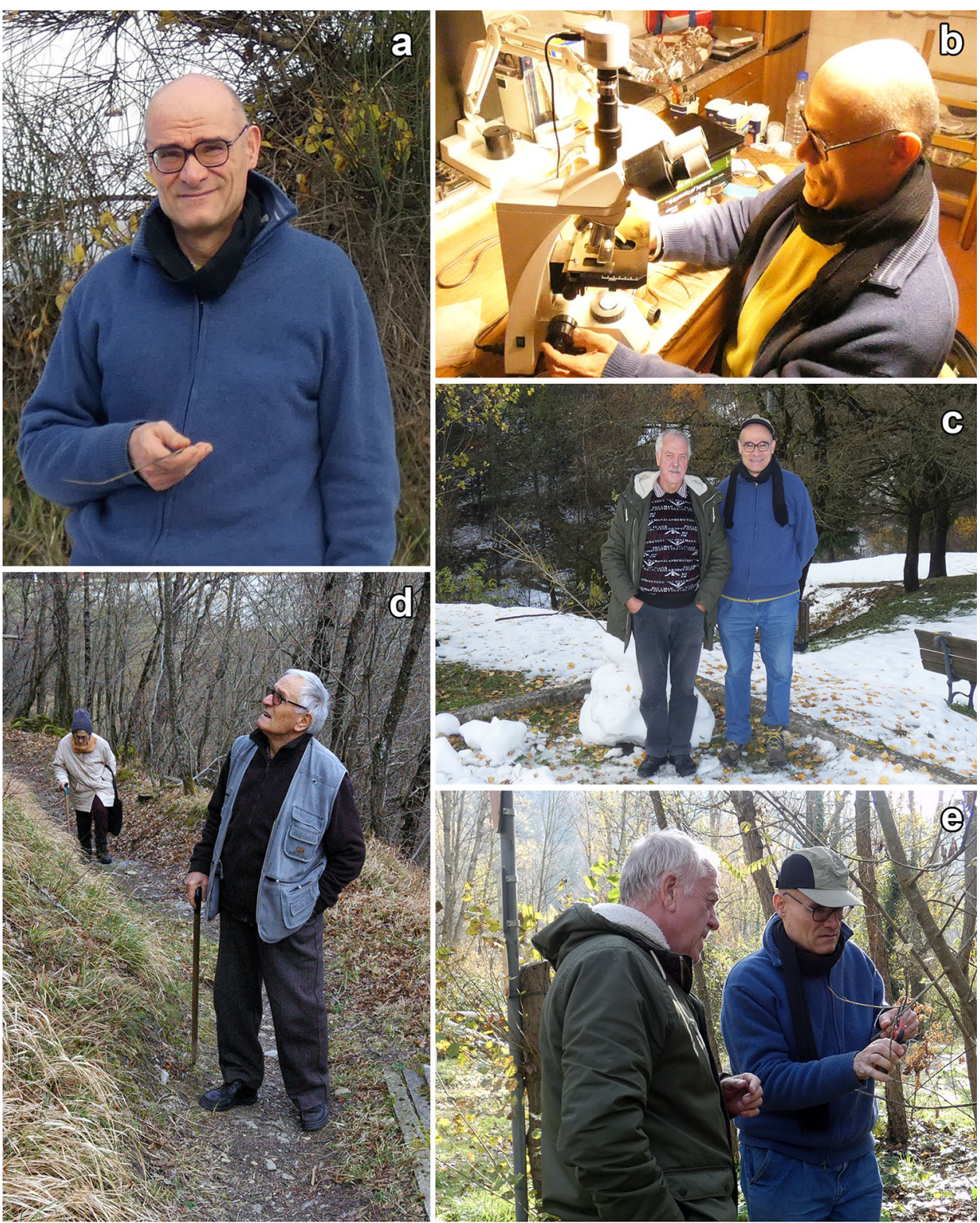

collection and study in Italy. Saccardo's collection contains almost 4500 fungal specimens, which are mostly assigned as type specimens of Ascomycota; these specimens were collected in Italy or sent to him from around the world. Therefore, modern fresh collections from Erio have proven invaluable for the advancement of studying ascomycetes.

Erio has collected more than 4500 fungal specimens from numerous plant genera, with plant identifications assisted by Giancarlo Lombardi, Gigi Stagioni and Sergio Montanari. According to his contributed collections, over 200 research articles that cite his specimens have been published with fellow mycologists and students. Ph.D. students have embraced his scientific collections with fungal illustrations for identification in academic research. Fungal diversity would not have advanced without these numerous resources. Several fungal species studied in the past centuries could not have been verified in the modern scientific sense without these interpretive specimens. Important epitype and neotype specimens and types of many novel genera and species have been discovered through his collections. The Ph.D. research of Hiran A. Ariyawansa, Dhanushka N. Wanasinghe, Indunil C. Senanayake, Wen-Jing Li, Chayanard Phukhamsakda and Nalin $\mathrm{N}$. Wijayawardene could not have been completed without Erio's significant contributions, while several other students have relied on his collections.

Erio's contribution to mycology has profoundly influenced the younger generation of mycologists. Thus, we would like to thank Erio for his work in promoting mycology and his collaboration as a great amateur 
Fig. 2 Some of the fungal paintings by Erio Camporesi. Used with permission
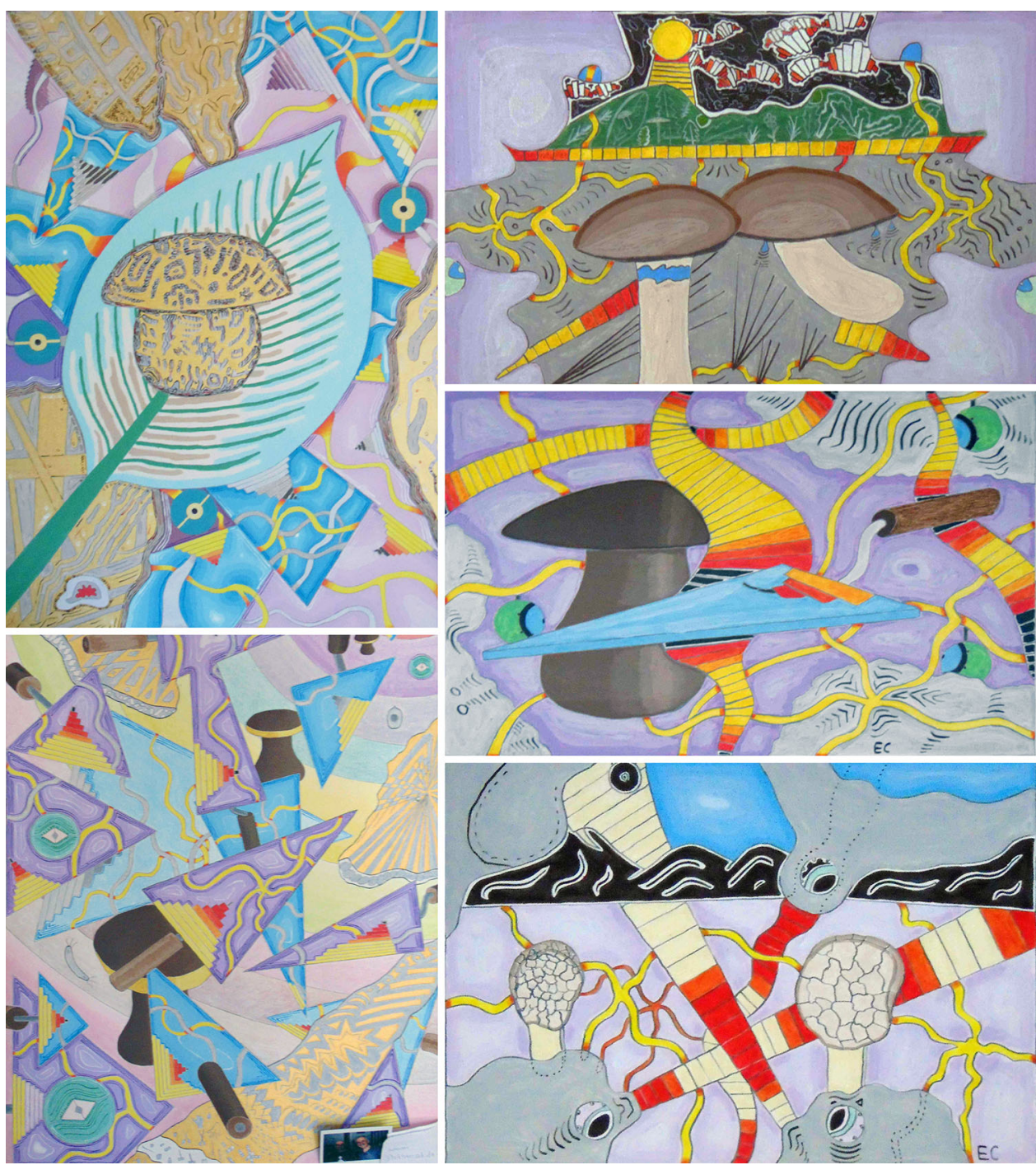

mycologist. We also would like to pass our sincere thanks to many other prominent amateur mycologists for their progress in mycological research, such as Alain Gardiennet, Christian Lechat, Jacques Fourier, Michel Hairaud, Hans-Otto Baral, Pedro Zapico and many others. This special issue is dedicated to the appreciation of amateur mycologists worldwide. Many of the recent significant accomplishments would not be possible without your continual encouragement and efforts.
This special issue is dedicated to Erio Campesori and other dedicated amateur mycologists for the tireless enthusiasm in collecting and studying fungi, which they do for fun and as a hobby. Some like Erio, have worked tirelessly with Ph.D. students and have provided a wealth of information on Italian microfungi. Thank you Erio, for your amazing efforts in providing material of fungi leading to numerous advancements in mycology, which otherwise would not have been possible. 